\title{
A jigsaw-puzzle imagery task for assessing active visuospatial processes in old and young people
}

\author{
JOHN T. E. RICHARDSON \\ Brunel University, Uxbridge, England \\ and \\ TOMASO VECCHI \\ University of Pavia, Pavia, Italy
}

\begin{abstract}
Recent studies have suggested a theoretical distinction between active elaboration and passive storage in visuospatial working memory, but research with older adults has failed to demonstrate a differential preservation of these two abilities. The results are controversial, and the investigation of the active component has been inhibited by the absence of any appropriate experimental procedures. A new task was developed involving the mental reconstruction of pictures of objects from fragmented pieces, and this provides a useful procedure for exploring active visuospatial processing. Significant differences in terms of both correctness and response latency were obtained between young and older adults and between younger old and older old adults. Performance also varied with visual complexity, mental rotation, and processing load. It is concluded that this ecologically relevant procedure constitutes a very powerful, sensitive, and reliable tool for identifying individual differences in visuospatial working memory.
\end{abstract}

The applied demands for the development of adequate instruments to assess memory functions are numerous. However, existing clinical and experimental tools are not always reliable, and they do not provide a clear link with theoretical expectations and empirical results. Moreover, tools for the investigation of visuospatial abilities are particularly poor. In the early 1970s, Corsi and Milner (Corsi, 1972; Milner, 1971) devised such a procedure (nowadays generally known as the Corsi block task), and this has long been regarded as the universal tool for assessing visuospatial memory span. Although it is widely used in clinical practice and neuropsychological assessment, the cognitive abilities underlying the execution of this task are still a matter of dispute (see Berch, Krikorian, \& Huha, 1998), and procedural variations can lead to variations in performance. The Corsi task is especially difficult to interpret in terms of a "working memory" system and more specifically in terms of a visuospatial working memory (VSWM) component that is devoted to the storage and processing of visuospatial material.

Over the last 20 years, many studies have investigated the structure of working memory (e.g., Baddeley, 1986; Bad-

The research reported in this paper was begun while T.V. was a visiting research fellow at Brunel University. The authors' subsequent collaboration was facilitated by a biomedical research collaboration grant from the Wellcome Trust (048699/Z/96). We are grateful to Cristina Alcaraz Andreu for her help in testing the participants in Experiment 3. Correspondence should be addressed to J. T. E. Richardson, Institute of Educational Technology, The Open University, Walton Hall, Milton Keynes MK7 6AA, England (e-mail: j.t.e.richardson@open.ac.uk). deley \& Hitch, 1974), which is thought to consist of a central executive plus a number of subsystems related to specific sensory modalities. The VSWM component or "visuospatial sketchpad" has been the least studied, and only in the last few years has the structure of this subsystem been investigated (see Cornoldi \& Vecchi, 2000; Logie, 1995). It is devoted to the storage and manipulation of visuospatial information, and some recent research has investigated the possibility that its functioning could be differentiated on the basis of the type of material involved (visual vs. spatial) and also separately on the basis of the type of processing required (passive storage vs. active processing). This distinction between visual and spatial processes is supported both by experimental findings (Logie \& Marchetti, 1991) and by neuropsychologicalevidence (Farah, Hammond, Levine, \& Calvanio, 1988; Luzzatti, Vecchi, Agazzi, Cesa-Bianchi, \& Vergani, 1998). However, it does not seem to capture the nature of the deficits that are shown by blind people in carrying out visuospatial tasks (Vecchi, 1998; Vecchi, Monticelli, \& Cornoldi, 1995).

More generally, studies investigating VSWM by means of an individual differences paradigm implicate the type of process involved as the chief variable determining participants' performance. The distinction between passive storage and active elaboration within VSWM has been posited in a number of theoretical accounts (e.g., Carpenter \& Just, 1989; Cornoldi \& Vecchi, 2000; Helstrup, 1989), and it has been confirmed in research into imagery in the blind, gender differences, and lifespan development. Contrary to intuition, several studies have demonstrated the ability of 
blind people to perform visuospatial tasks (e.g., Cornoldi, Cortesi, \& Preti, 1991; Kerr, 1983; Marmor \& Zaback, 1976; Vecchi et al., 1995). If they are presented with a task requiring them to memorize the position of different objects within a spatial array, they perform at a similar level to that of sighted individuals. However, if they are required to transform, integrate, or modify visuospatial material, their performance drops significantly (Cornoldi, Bertuccelli, Rocchi, \& Sbrana, 1993; Vecchi, 1998; Vecchi et al., 1995).

Several studies have suggested a male superiority in visuospatial tasks (e.g., Maccoby \& Jacklin, 1974), but a more detailed analysis shows that this advantage is related to sociocultural factors rather than sexual differences (see, e.g., Caplan, Crawford, Hyde, \& Richardson, 1997). Moreover, the direction of the gender difference depends upon specific properties of the task being performed (Paivio \& Clark, 1991). Harshman and Paivio (1987; Paivio \& Harshman, 1983) reported that men performed better than women in tasks requiring the rotation or transformation of mental images, but the opposite was true for passive recall or vividness-rating tasks. A similar result was obtained by Harshman, Hampson, and Berenbaum (1983): Despite the male superiority in mental rotation, transformation, or spatial relation, women performed better in the recognition of figures or in mental comparison tasks. Gender differences were recently investigated in a paradigm addressing the passive-active distinction showing that men and women performed at the same level in the recall of spatial configurations, but a clear difference in favor of male participants was found in the elaboration of a pathway through spatial coordinates (Vecchi \& Girelli, 1998).

Studies of the development of VSWM over the normal lifespan have also implicated the amount of visuospatial elaboration as a key variable determining increasing performance in child development and declining performance in older adults. The ability of young children to use visual imagery has frequently been reported (e.g., Hitch, Woodin, \& Baker, 1989; Kosslyn, Margolis, Barrett, Goldknopf, \& Caly, 1990), and Kosslyn (1980) suggested that they do so more extensively than adults. However, the quality of their images changes with age toward an increasing capacity for elaborating visuospatial material. In particular, the ability to transform, integrate, and modify visuospatial images develops between the ages of 8 and 14 (Kosslyn et al., 1990; Swanson, 1996). The development of active processing capacity in VSWM seems to be related to the ability to use spatial coordinates such as "right" and "left" (Roberts \& Aman, 1993), and this ability develops during the same period (Corballis \& Beale, 1976).

Evidence for a similar dissociation in older people is not so straightforward. Although a significant decline in visuospatial performance has been widely reported (for reviews, see Craik \& Salthouse, 1992; Salthouse, 1992), the specific nature of this deficit is still a matter of debate. The role of one specific factor affecting cognitive performance in older adults - namely, speed of processing - has been recently documented (Salthouse, 1994a; 1996; Salthouse \& Coon, 1993). This implies that reduced performance in older people should be more evident in active tasks. Although few studies have investigated this notion of a selective deficit in older people, initial results support the hypothesis that the aging process is associated with a deficit that is more evident in tasks that require the active processing of visuospatial information (Morris, Gick, \& Craik, 1988; Salthouse \& Mitchell, 1989).

Recently, Mayr and Kliegl (1993; Mayr, Kliegl \& Krampe, 1996) have suggested a different theoretical approach to investigate these aspects of visuospatial processing. These researchers postulated the existence of two different aspects of complexity (namely, sequential and coordinative complexity) that could be selectively affected in older people's performance. Sequential complexity, defined as "the speed of simple information processing steps," is affected by the reduced speed of processing, whereas coordinative complexity, defined as "the coordination of processing steps in working memory," is related to a specific decrement in the capacity to maintain, integrate, and transform different items in working memory (p. 1297). Mayr and Kliegl reported a dissociation between these two abilities in the aging process, thus challenging the idea that speed of processing was the only factor affecting performance.

The evidence that has so far been reported indicates that it is possible to differentiate among visuospatial abilities on the basis of the type of processes involved, and the different theoretical approaches agree in identifying tasks requiring the integration, coordination, or manipulation of different materials as being more sensitive to individual differences and especially to cognitive deterioration in old age. This subsumes the distinctions between passive and active processing (Cornoldi \& Vecchi, 2000; Vecchi, 1998), between structural and operational capacities (Salthouse \& Mitchell, 1989), and between sequential and coordinative complexities (Mayr \& Kliegl, 1993). Nevertheless, not all the empirical findings support this distinction. Salthouse, Babcock, and Shaw (1991) were unable to replicate the results obtained by Salthouse and Mitchell (1989): Age differences were reported on tasks measuring both passive storage and active processing (or both "structural" and "operational" capacities, on their definitions), and sometimes were not significant at all. In these tasks, the researchers used a computer-based procedure that may have been overly complex or overly artificial, and this may have affected their findings: To assess the structural capacity of working memory, the participants had to memorize stimuli presented in the four quadrants of a computer screen; in addition, they were asked to perform appropriate processing operations (verbal or spatial) on the basis of the stimuli that had appeared in just one of the quadrants.

One possibility is that this task was simply too complex, and this would specifically have affected some of the experiments in which no differences were obtained between young and older adults. Moreover, the overall complexity of passive tasks in which the participants had to store large amounts of information could have demanded the use of additional strategies (such as verbal rehearsal) to perform 
the task. If this is so, then the passive task might have tapped some form of active processing. Alternatively, active elaboration could be related to some kind of passive storage in order to remember the results of successive mental operations. The choice of the particular tasks used in different experiments could be a critical determinant of the findings obtained, and this is likely to be particularly evident if experimental procedures are used with people who have already experienced some reduction in their cognitive functions, such as older adults. Although empirical procedures are available to study the passive store (e.g., the visual pattern test: Della Sala, Gray, Baddeley, \& Wilson, 1997), the active elaboration component has been investigated only fairly recently, and the lack of appropriate experimental techniques has often inhibited empirical investigations. The experiments that are reported here were not intended to resolve the debate over whether active or passive visuospatial processes are differentially affected by age, but instead and more specifically to provide information on the functioning of active processes and the variables that might affect their capacity.

We have endeavored to develop an appropriate procedure for analyzing active visuospatial processing. We have also considered the ecological value of the task as another variable potentially affecting the results. Seeking a task that could be used with older adults and in the future as a tool for neuropsychological assessment, we have developed a methodology that possesses good ecological validity and also appears to be sensitive in identifying individual differences (Vecchi \& Richardson, 2000). The participants are asked to solve jigsaw puzzles consisting of four, six, or nine pieces without actually touching or moving the pieces in question (Figure 1). Each piece is numbered, the partic- ipants are given a prepared response sheet showing the original outline of the completed puzzle, and they are instructed to write down the numbers corresponding to the pieces in the correct spatial positions. Performance is evaluated in terms of the percentage of correct responses as well as in terms of the time needed to solve each of the puzzles.

The stimuli were all pictures of inanimate objects chosen from the standardized material constructed by Snodgrass and Vanderwart (1980). To facilitate recognition of the objects and to obtain the purest active visuospatial elaboration, all of the stimuli had high values in terms of rated familiarity (defined by Snodgrass and Vanderwart as "the degree to which people come into contact with or think about the concept") and image agreement ("how closely each picture resembles people's mental image of the object"). Stimuli were chosen to represent five levels of visual complexity ("the amount of detail or intricacy of line"), thus enabling us to evaluate the role of visual complexity in the elaboration of visuospatial images. Superficially, this task is similar to other tests that have been used in the past to assess visuospatial abilities. However, our task taps a number of distinct functions and is of particular relevance to current discussions concerning VSWM.

\section{EXPERIMENT 1}

In this investigation, we evaluated the effects of processing load, visual complexity, and (in Experiment 2 below) mental rotation. In principle, these variables represent three different kinds of complexity with regard to (1) the number of units that the system can process at any one time (number of pieces), (2) the properties of the object itself (overall visual complexity), and (3) the properties of the
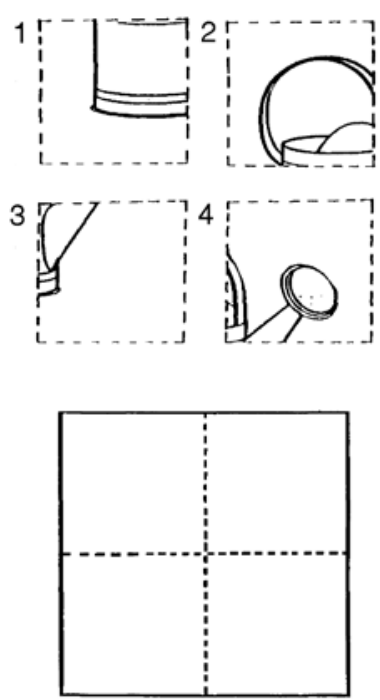

Figure 1. Examples of puzzles containing four, six, and nine pieces (watering can, toaster, and telephone, respectively). The appropriate response sheet was presented at the same time, and the participants had to write down the correct numbers in the correct spatial locations. 
pieces (rotation), respectively. This test was administered to young adults, old adults aged roughly $60-75$, and old adults aged roughly $75-90$.

\section{Method}

Participants. Thirty-two healthy older adults were recruited through an advertisement placed in the newsletter produced by the London branch of the University of the Third Age. They were divided into two equal groups by age. The "younger old" group consisted of people between 60 and 75 years old (mean age $=68.9$ ), and the "older old" group consisted of people between 75 and 88 years old (mean age $=79.3$ ); all of these participants had a minimum of 11 years of education. Sixteen students taking courses at Brunel University were also recruited to participate in this experiment as a comparison group of young adults, in return for a monetary payment; they were between 18 and 27 years old (mean age $=20.7$ years). The three groups of participants were matched for sex and type of education/ profession. In addition, we excluded occupational groups that previous research had shown to be superior in performing visuospatial tasks, such as architects or taxi drivers (e.g., Salthouse, 1991; Salthouse, Babcock, Skovronek, Mitchell, \& Palmon, 1990). No participant took part in more than one of the experiments described in this paper.

Materials. Five sets of three pictures were chosen from the stimuli used by Snodgrass and Vanderwart (1980) to represent different levels of visual complexity along a rating scale from 1 to 5 . The 15 pictured objects and their relative normative values on visual complexity, familiarity, and image agreement are listed in the Appendix. The five sets of pictured objects did not vary significantly in either familiarity or image agreement ( $F<1$ in both cases). Across the set of 15 objects, image agreement showed a slight positive correlation with visual complexity $(r=+.24)$, whereas familiarity showed slight negative correlations with both visual complexity $(r=-.19)$ and image agreement $(r=-.17)$. None of these correlation coefficients even approached statistical significance ( $p>.30$ in each case).

Each of the pictures was fragmented into four, six, and nine numbered pieces to produce 45 different puzzles. The pictures were enlarged to fit an area $15 \mathrm{~cm} \times 15 \mathrm{~cm}$ and were then divided into four pieces $7.5 \mathrm{~cm} \times 7.5 \mathrm{~cm}$, six pieces $5 \mathrm{~cm} \times 7.5 \mathrm{~cm}$, or nine pieces $5 \mathrm{~cm}$ $\times 5 \mathrm{~cm}$. The fragments of each picture were displayed on a separate sheet of paper in the correct orientation but in a jumbled configuration. Each participant was shown 15 puzzles representing all possible combinations of visual complexity and number of pieces, but the number of pieces for the three stimuli at each level of visual complexity was roughly counterbalanced across participants. Puzzles representing the 15 stimuli were shown in six different combinations of pictures and fragmentation. The prepared response sheets contained grids of the same size as the original pictures and divided into the appropriate number of squares. Two further practice puzzles were prepared and were presented at the beginning of the experimental session.

Procedure. The participants were tested individually, seated in front of the experimenter. They were given a fragmented picture together with an appropriate response sheet, and the name of the object in question was read out loud by the experimenter. They then had to write down the appropriate numbers in the correct spatial locations on the prepared response sheet. The participants were allowed to correct their errors within the time limit if they wished. We recorded the accuracy of the solutions the participants achieved in $3 \mathrm{~min}$; if participants indicated that they were satisfied with their solution within the time limit, we also recorded the latency. When the puzzle had been solved or the time limit had been reached, the correct solution was presented by way of feedback and to avoid interference with successive pictures before the next puzzle was presented. The entire experiment lasted for approximately $30 \mathrm{~min}$.

\section{Results and Discussion}

We analyzed participants' performance on each trial in terms of the percentage of pieces placed in their correct spatial locations and in terms of the percentage of correct links between the pieces regardless of their absolute locations. In principle, these two measures could reflect different strategies used by the participants; in practice, however, they revealed very similar patterns of performance, and so only the analysis of correct locations will be described in detail. Table 1 summarizes the means and standard deviations for the percentage of correct locations, the percentage of correct links, and the solution times.

An analysis of variance (ANOVA) was carried out on the percentage of correct locations, using the betweensubjects factor of age (young, younger old, and older old) and the within-subjects factors of visual complexity and processing load (four, six, and nine pieces). All the main effects and two-way interactions were statistically significant: age $\left[F(2,45)=18.52, M S_{\mathrm{e}}=0.49, p<.001\right]$, visual complexity $\left[F(4,180)=15.30, M S_{\mathrm{e}}=0.05, p<.001\right]$, processing load $\left[F(2,90)=58.83, M S_{\mathrm{e}}=0.06, p<.001\right]$, age $\times$ visual complexity $\left[F(8,180)=3.78, M S_{\mathrm{e}}=0.05, p<.001\right]$ and age $\times$ processing load $\left[F(4,90)=7.66, M S_{\mathrm{e}}=0.06\right.$,

Table 1

Mean Values (and Standard Deviations) for Percentage of Correct Locations, Percentage of Correct Links, and Response Latencies (in Seconds) for Young Adults, Younger Old Adults, and Older Old Adults Solving Puzzles Containing Four, Six, and Nine Pieces (Experiment 1)

\begin{tabular}{|c|c|c|c|c|c|c|}
\hline & \multicolumn{2}{|c|}{ Young } & \multicolumn{2}{|c|}{ Younger Old } & \multicolumn{2}{|c|}{ Older Old } \\
\hline & $M$ & $S D$ & $M$ & $S D$ & $M$ & $S D$ \\
\hline \multicolumn{7}{|l|}{ Locations } \\
\hline Four pieces & 94.7 & 6.7 & 81.9 & 16.6 & 71.3 & 24.2 \\
\hline Six pieces & 94.4 & 11.6 & 73.1 & 28.0 & 53.4 & 25.1 \\
\hline Nine pieces & 87.3 & 13.6 & 49.6 & 24.8 & 36.4 & 21.0 \\
\hline \multicolumn{7}{|l|}{ Links } \\
\hline Four pieces & 92.5 & 11.1 & 77.8 & 20.8 & 69.1 & 26.3 \\
\hline Six pieces & 92.1 & 15.9 & 64.6 & 30.6 & 44.3 & 26.5 \\
\hline Nine pieces & 82.4 & 18.6 & 39.9 & 22.6 & 26.7 & 20.1 \\
\hline \multicolumn{7}{|l|}{ Latencies } \\
\hline Four pieces & 9.7 & 5.5 & 28.2 & 14.1 & 44.5 & 24.1 \\
\hline Six pieces & 25.5 & 9.3 & 71.9 & 29.0 & 95.2 & 33.5 \\
\hline Nine pieces & 72.8 & 17.2 & 131.4 & 29.8 & 145.4 & 33.0 \\
\hline
\end{tabular}


$p<.005]$. The three-way interaction was not significant $\left[F(16,360)=1.13, M S_{\mathrm{e}}=0.05, p>.30\right]$.

Table 1 shows that performance was poorer in the older adults than in the young adults and poorer in the older old adults than in the younger old adults. Performance was inversely related to processing load, and this was more evident in the two older groups because of a ceiling effect in the young adults. A posteriori tests showed that the effect of processing load was highly significant for the younger old adults $[F(2,90)=34.23, p<.001]$ and for the older old adults $[F(2,90)=37.64, p<.001]$, but not for the young adults $[F(2,90)=2.25, p>.10]$.

Figure 2 shows the effect of visual complexity for the three groups of participants. The effect was U-shaped; increasing complexity generally resulted in poorer performance except at the highest level of complexity. The latter facilitation appears to have arisen because the correct links between items become perceptually more obvious in the case of the highly complex stimuli. The effect of visual complexity varied with the processing load, but it was statistically significant for problems containing four, six, or nine pieces. Conversely, the effect of processing load was statistically significant at all levels of complexity.

The effect of visual complexity was highly significant for the older old adults $[F(4,180)=19.69, p<.001]$, just significant for the younger old adults $[F(4,180)=2.44, p=$ $.05]$, and not significant at all for the young adults $(F<1)$. The fact that this effect was apparent mainly in the older old group suggests that it was due to a selective overload of the system capacity, possibly requiring the use of additional (perceptual) strategies.

A separate ANOVA was carried out on the solution times, using the same design. All the main effects were statistically significant: age $\left[F(2,45)=35.42, M S_{\mathrm{e}}=6,209.57\right.$, $p<.001]$, visual complexity $\left[F(4,180)=7.52, M S_{\mathrm{e}}=\right.$ $771.54, p<.001]$, and processing load $[F(2,90)=405.99$, $\left.M S_{\mathrm{e}}=1,186.07, p<.001\right]$. The interaction between the effects of age and processing load was also significant $\left[F(4,90)=9.98, M S_{\mathrm{e}}=1,186.07, p<.001\right]$. No other term in this analysis was statistically significant.

Table 1 shows that, as expected, the solution time increased with the number of pieces. The interaction with age was due to a floor effect on the four-piece problems in the young adults. Nevertheless, a posteriori tests showed that the effect of processing load was highly significant for the young adults $[F(2,90)=72.89]$, the younger old adults $[F(2,90)=181.13]$, and the older old adults $[F(2,90)=$ $171.93, p<.001]$ in each case. The effect of visual complexity was analogous to that obtained in the case of the percentage of correct locations, with visual complexity generally resulting in slower performance except at the highest level of complexity.

In this experiment, different stimuli were presented to different participants in different conditions. This means that it is not possible to compute a measure of the internal consistency of the task, such as Cronbach's coefficient alpha. However, Winer, Brown, and Michels (1991, pp. 10171018) described a technique for estimating the test-retest reliability of measurements obtained using a series of instruments. This compares the between-subjects error term and the pooled within-subjects error term in a repeated measures ANOVA, which removes the contribution of the anchor point (or main effect) of each instrument. Applying Winer et al.'s technique to the data from the present experiment, the estimated reliability of each participant's score on each puzzle was .3546 , and the estimated reliability of each participant's mean score across all of the 15 jigsaw puzzles was .8918. Hence, the jigsaw-puzzle task provides a highly reliable way of measuring active visuospatial processing in participants of different ages.

The significant effects of processing load upon both the percentage of correct locations and the solution time imply that a limited-capacity VSWM is being employed in this situation. The pattern of results is consistent with previous findings, which indicate a cutoff for young adults between six and nine processing units (Vecchi \& Richardson, 2000). However, older people show a limited processing capacity that interacts (to some extent, at least) with the use of perceptual aids, as shown by the visual complexity effect. The use of a perceptual strategy and the related visual complexity effect could be interpreted as a symptom of limited capacity for active processing. In Experiment 1 , only the older old group experienced an overload of the system, although all the participants were af-

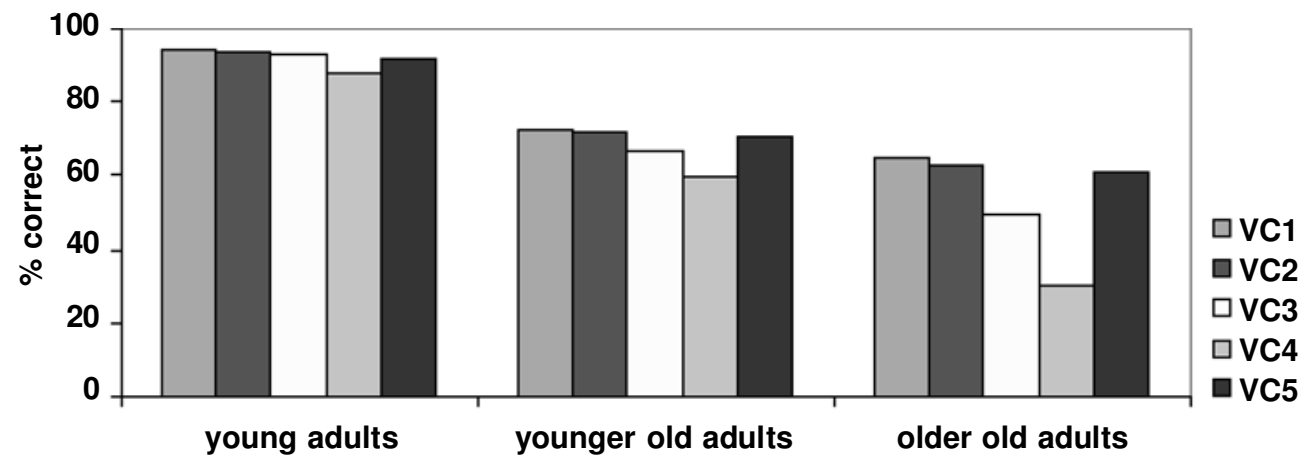

Figure 2. Mean percentage of correct locations for target stimuli of five levels of visual complexity (Experiment 1). 
fected to some extent by increasing complexity. If our hypothesis is correct, the visual complexity effect should be obtained in younger people, too, in more complex and demanding tasks. To test this theoretical inference, we increased the complexity of our task by introducing the factor of mental rotation.

\section{EXPERIMENT 2}

In this experiment, we extended the general procedure to incorporate an additional processing load in the form of mental rotation while attempting to retain the ecological nature of the task. The participants were asked to solve the same puzzles as in Experiment 1, but in each case roughly half of the pieces were presented upside-down and would therefore have to be mentally rotated to derive a correct solution. We chose this in preference to the situation where all of the fragments were presented upside-down because our previous studies had shown the former to be more complex and more sensitive in detecting individual differences (Vecchi \& Richardson, 2000).

\section{Method}

Participants. Thirty-two healthy older participants were divided into two equal groups on the basis of their age; they were again recruited through the London branch of the University of the Third Age, and all had a minimum of 11 years of education. The younger old group consisted of people between 60 and 73 years old (mean age $=67.4$ ), and the older old group consisted of people between 77 and 90 years old (mean age $=82.6$ ). Sixteen students from Brunel University participated in this experiment as a group of young adults in return for a monetary payment; they were between 17 and 30 years old (mean age $=20.7$ ). The three groups of participants were again matched for sex and type of education/profession.

Materials and Procedure. The materials and procedure were identical to those in Experiment 1, except that roughly half of the pieces in each puzzle (i.e., two, three, or four pieces in the case of puzzles consisting of four, six, and nine pieces, respectively) were presented rotated through $180^{\circ}$. The entire experiment lasted about $35 \mathrm{~min}$.

\section{Results and Discussion}

As in Experiment 1, similar patterns of performance were obtained in the analyses of correct locations and correct links, and only the analysis of correct locations will be described in detail below. Table 2 summarizes the means and standard deviations for the percentage of correct locations, the percentage of correct links, and the solution times. An ANOVA was carried out on the percentage of correct locations, using the between-subjects factor of age (young, younger old, and older old) and the within-subjects factors of visual complexity and processing load (four, six, and nine pieces).

All of the main effects were once again statistically significant: age $\left[F(2,45)=37.49, M S_{\mathrm{e}}=0.43, p<.001\right]$, visual complexity $\left[F(4,180)=12.79, M S_{\mathrm{e}}=0.06, p<.001\right]$, and processing load $\left[F(2,90)=45.98, M S_{\mathrm{e}}=0.07, p<.001\right]$. There was a significant interaction between the effects of visual complexity and processing load $[F(8,360)=2.65$, $\left.M S_{\mathrm{e}}=0.08, p<.01\right]$, but there was no significant interaction between the effects of age and visual complexity $\left[F(8,180)=1.59, M S_{\mathrm{e}}=0.06\right]$ or between the effects of age and processing load $\left[F(4,90)=1.61, M S_{\mathrm{e}}=0.07, p>.10\right.$ in each case], and the three-way interaction was not significant $(F<1)$.

Table 2 shows that performance was again poorer in the older adults than in the young adults and poorer in the older old adults than in the younger old adults. Performance was inversely related to processing load, and this was apparent in each of the three groups. Figure 3 shows the effect of visual complexity in the three groups of participants. Once again, increasing complexity generally resulted in poorer performance, except at the highest level of complexity, and this pattern, too, was evident in all three groups. The effect of processing load depended upon visual complexity, partly because of a ceiling effect in $\mathrm{VC} 1$ stimuli and a floor effect in VC4 stimuli. However, a posteriori tests showed that the main effect of visual complexity was significant for puzzles with four, six, and nine pieces, and that the effect of processing load was significant at each level of visual complexity.

A separate ANOVA was carried out on the solution times, using the same design. In this experiment, all the main effects and two-way interactions were statistically significant:age $\left[F(2,45)=16.16, M S_{\mathrm{e}}=11,046.48, p<.001\right]$,

Table 2

Mean Values (and Standard Deviations) for Percentage of Correct Locations, Percentage of Correct Links, and Response Latencies (in Seconds) for Young Adults, Younger Old Adults, and Older Old Adults Solving Puzzles Containing Four, Six, and Nine Pieces (Experiment 2)

\begin{tabular}{|c|c|c|c|c|c|c|}
\hline & \multicolumn{2}{|c|}{ Young } & \multicolumn{2}{|c|}{ Younger Old } & \multicolumn{2}{|c|}{ Older Old } \\
\hline & $M$ & $S D$ & $M$ & $S D$ & $M$ & $S D$ \\
\hline \multicolumn{7}{|l|}{ Locations } \\
\hline Four pieces & 86.9 & 11.8 & 61.9 & 24.6 & 37.2 & 19.9 \\
\hline Six pieces & 80.2 & 14.6 & 43.8 & 30.7 & 29.0 & 16.2 \\
\hline Nine pieces & 68.5 & 16.4 & 30.0 & 23.1 & 15.8 & 11.6 \\
\hline \multicolumn{7}{|l|}{ Links } \\
\hline Four pieces & 84.7 & 12.3 & 54.1 & 28.6 & 27.8 & 24.1 \\
\hline Six pieces & 72.9 & 19.4 & 36.6 & 27.7 & 21.8 & 13.9 \\
\hline Nine pieces & 60.4 & 17.6 & 25.4 & 21.9 & 8.2 & 9.1 \\
\hline \multicolumn{7}{|l|}{ Latencies } \\
\hline Four pieces & 25.2 & 13.0 & 32.8 & 15.3 & 73.5 & 40.4 \\
\hline Six pieces & 61.3 & 31.7 & 79.7 & 27.8 & 127.1 & 43.0 \\
\hline Nine pieces & 110.6 & 30.6 & 134.0 & 33.6 & 156.3 & 31.0 \\
\hline
\end{tabular}


visual complexity $\left[F(4,180)=10.14, M S_{\mathrm{e}}=840.76, p<\right.$ $.001]$, processing load $\left[F(2,90)=283.80, M S_{\mathrm{e}}=1,705.78\right.$, $p<.001]$, age $\times$ visual complexity $\left[F(8,180)=3.93, M S_{\mathrm{e}}=\right.$ $840.76, p<.001]$, age $\times$ processing load $[F(4,90)=2.77$, $\left.M S_{\mathrm{e}}=1,705.78, p<.05\right]$, and visual complexity $\times$ processing load $\left[F(8,360)=2.51, M S_{\mathrm{e}}=771.02, p<.02\right]$. However, the three-way interaction was not statistically significant $\left[F(16,360)=1.49, M S_{\mathrm{e}}=771.02, p>.10\right]$.

Table 2 shows that the solution time once again increased with the number of pieces, and the interaction with age was mainly due to the solution times on the ninepiece problems approaching the upper limit of $3 \mathrm{~min}$ in the case of the older old adults. Nevertheless, a posteriori tests showed that the effect of processing load was highly significant in the young adults $[F(2,90)=86.23]$, in the younger old adults $[F(2,90)=120.30]$, and in the older old adults $[F(2,90)=82.81, p<.001]$ in all three cases.

The effect of visual complexity upon solution time was once again analogous to that obtained in the case of the percentage of correct locations, with solution time increasing with visual complexity except for the most complex stimuli. A posteriori tests showed that the main effect of visual complexity was highly significant in the young adults $[F(4,180)=11.36, p<.001]$ and in the younger old adults $[F(4,180)=6.18, p<.001]$, but was not at all significant in the older old adults $(F<1)$. In the latter group, mean solution times tended to approach the upper limit of $3 \mathrm{~min}$ at all five levels of visual complexity. Finally, the effect of visual complexity varied with processing load. A posteriori tests showed that it was not significant for four-piece problems $[F(4,540)=2.10, p>.05]$, significant for six-piece problems $[F(4,540)=2.90, p<.025]$, and highly significant for nine-piece problems $[F(4,540)=10.60, p<.001]$. Nevertheless, the effect of processing load was highly significant at all five levels of visual complexity.

In Experiment 2, the estimated reliability of each participant's score on each puzzle was .2529 , and the estimated reliability of each participant's mean score across all 15 puzzles was .8355 . Once again, this task proved to be a highly reliable way of measuring active visuospatial processing in participants of different ages.

\section{EXPERIMENT 3}

Experiments 1 and 2 showed the adequacy and reliability of this test when employed to measure individual differences in visuospatial abilities. Moreover, variations in processing load significantly affected participants' performance, thus indicating the sensitivity of this test and the possibility of using it as a clinical or an experimental tool. Experiment 3 was designed to obtain more information about the cognitive abilities underlying the execution of this task.

The characteristics of the task were investigated by means of a dual-task paradigm using three interfering tasks that are often employed to investigate the structure of working memory: articulatory suppression (to disrupt the verbal working memory component), tapping (to disrupt the visuospatial working memory component), and random generation (to disrupt the amodal central executive component). Once again, we tested a group of young adults and two groups of older adults. One of the items used for practice in the two previous experiments was added to the experimental items, yielding a set of 16 pictures. These were reclassified into four sets that were roughly matched in terms of mean visual complexity. These four sets were then presented in a baseline condition (no interference) as well as in the three interference conditions.

\section{Method}

Participants. Thirty-two healthy older participants with at least 11 years of education were recruited through the Pavia branch of the University of the Third Age. They were divided into two equal groups on the basis of their age: The younger old group consisted of people between 60 and 68 years old (mean age $=63.3$ ), and the older old group consisted of people between 70 and 75 years old (mean age $=71.8$ ). Sixteen students and technicians from the University of Pavia volunteered to participate in this experiment as a group of young adults; they were between 30 and 39 years old (mean age = 34.4 years). Once again, the three groups of participants were matched for sex and type of education/profession.

Materials and Procedure. Sixteen pictures were chosen from those used in the previous experiments. The suitcase was added to the experimental stimuli to obtain four sets of four pictures of approximately equal visual complexity: The mean visual complexity values of the four sets of stimuli were $3.07,3.14,3.15$, and 3.24. All

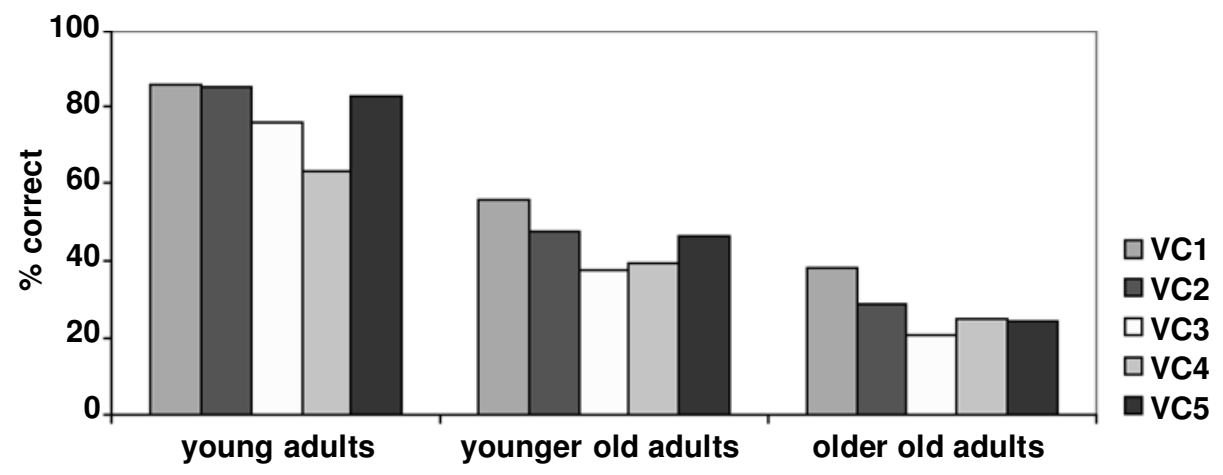

Figure 3. Mean percentage of correct locations for target stimuli of five levels of visual complexity (Experiment 2). 
of the pictures were presented in the nine-piece condition and in the correct orientation (as in Experiment 1). The general procedure was identical to that already described, except that the time limit was fixed at $150 \mathrm{sec}$.

In addition, except for the baseline condition (which was identical to the previous experiments), the participants were required to perform an interfering task while solving the puzzles: (1) random generation (central interference) - the participants were instructed to produce random sequences of letters by saying them aloud at a rate of 1 letter/sec; (2) articulatory suppression (verbal interference) the participants were instructed to say aloud continuously the syllable "la, la, la..." at a rate of 2 syllables/ sec; (3) tapping (visuospatial interference) - the participants were instructed to use their nonpreferred hands to touch in sequence the four corners of a $3 \times 3$ matrix printed on a sheet of paper at a rate of 2 taps/sec.

The order of presentation of these four conditions (the baseline and three interfering conditions) was counterbalanced following a Latin-square design, thus yielding four different orders. However, the pictures themselves were always presented in the same order: What varied was their association with each specific experimental condition. Thus, the combination of picture set and type of interference was counterbalanced across participants. The clock was used as practice trial and presented at the beginning of each experimental condition associated with the different types of interference. The entire experiment lasted approximately $30 \mathrm{~min}$.

\section{Results and Discussion}

Table 3 summarizes the means and standard deviations for the percentage of correct locations and the solution times. Performance was poorer in the older adults than in the young adults and poorer in the older old than in the younger old adults. However, all the participants showed similar effects of interference, indicating that aging did not differentially influence the cognitive structures underlying the execution of this task. An ANOVA was carried out on the percentage of correct locations, using the betweensubjects factor of age (young, younger old, and older old) and the within-subjects factor of interference (baseline and central, verbal, or visuospatial interference).

This produced a significant main effect of age $[F(2,45)=$ $\left.38.13, M S_{\mathrm{e}}=354.15, p<.001\right]$. The main effect of interference approached significance $\left[F(3,135)=2.42, M S_{\mathrm{e}}=\right.$ $65.57, p<.07]$, but the interaction between the two variables was not significant $\left[F(6,135)=1.51, M S_{\mathrm{e}}=65.57\right.$, $p>.15]$. A posteriori tests carried out to investigate the ef- fects of age using Tukey's procedure revealed significant differences among all possible pairs of groups: The young adults outperformed the younger old adults, who in turn outperformed the older old adults. A posteriori tests carried out to investigate the effects of interference using Dunnett's (1955) method revealed that both central interference and visuospatial interference gave rise to a significant reduction in performance in comparison with the baseline ( $p<.05$ in both cases), but that verbal interference did not.

A separate ANOVA was carried out on the solution times, using the same design. This produced significant main effects of age $\left[F(2,45)=50.41, M S_{\mathrm{e}}=2,973.03, p<.001\right]$ and of interference $\left[F(3,135)=14.26, M S_{\mathrm{e}}=176.32, p<\right.$ $.001]$. The interaction between the two variables was again not significant $[F(6,135)=1.87, p>.05]$. A posteriori tests carried out to investigate the effects of age using Tukey's procedure revealed significant differences among all possible pairs of groups: The young adults performed more quickly than the younger old adults, who in turn performed more quickly than the older old adults. A posteriori tests carried out to investigate the effects of interference using Dunnett's (1955) method showed that central interference and visuospatial interference gave rise to significantly slower performance in comparison with the baseline $(p<.01$ in both cases $)$, but that verbal interference did not.

One limitation of the dual-task procedure used in this experiment was that participants in the visuospatial interference condition were required to tap and write their responses at the same time. It is possible that part of the effect of concurrent tapping upon performance in the jigsawpuzzle task could have arisen at a purely peripheral level, particularly in terms of the participants' increased solution times. On the face of it, this problem appears to be insoluble using dual-task methodology, since it is hard to think of a concurrent visuospatial task that is observable but does not involve either visual input or motor responses (and would thus avoid peripheral forms of interference). Our final experiment adopted a different methodology, the individual differences approach, to study the processing demands of the jigsaw-puzzle task.

Table 3

Mean Values (and Standard Deviations) for Percentage of Correct Locations and Response Latencies (in Seconds) for Young Adults, Younger Old Adults, and Older Old Adults Solving Puzzles Under a Baseline Condition and Three Interference Conditions (Experiment 3)

\begin{tabular}{|c|c|c|c|c|c|c|c|c|}
\hline & \multicolumn{2}{|c|}{ Young } & \multicolumn{2}{|c|}{ Younger Old } & \multicolumn{2}{|c|}{ Older Old } & \multicolumn{2}{|c|}{ Overall } \\
\hline & $M$ & $\overline{S D}$ & $M$ & $S D$ & $M$ & $S D$ & $M$ & $S D$ \\
\hline \multicolumn{9}{|l|}{ Locations } \\
\hline Baseline & 98.9 & 2.8 & 84.6 & 12.2 & 75.7 & 15.0 & 86.5 & 14.6 \\
\hline Central interference & 97.9 & 3.4 & 81.2 & 13.2 & 67.9 & 18.3 & 82.3 & 17.8 \\
\hline Visuospatial interference & 97.9 & 4.8 & 82.2 & 10.1 & 68.4 & 14.9 & 82.9 & 16.1 \\
\hline Verbal interference & 99.7 & 1.0 & 85.1 & 7.2 & 66.1 & 18.5 & 83.7 & 17.8 \\
\hline \multicolumn{9}{|l|}{ Latencies } \\
\hline Baseline & 20.1 & 7.9 & 69.2 & 39.4 & 116.3 & 25.6 & 68.6 & 48.0 \\
\hline Central interference & 31.9 & 15.1 & 95.2 & 45.0 & 127.4 & 35.2 & 84.9 & 52.1 \\
\hline Visuospatial interference & 29.7 & 13.3 & 90.6 & 38.0 & 123.5 & 25.4 & 81.3 & 47.6 \\
\hline Verbal interference & 19.4 & 7.4 & 86.7 & 37.9 & 117.0 & 30.9 & 74.4 & 49.8 \\
\hline
\end{tabular}




\section{EXPERIMENT 4}

As we acknowledged earlier, our task shares certain superficial similarities with other tasks that have been used in the past to assess visuospatial abilities; these include Hooper's (1958) Visual Organization Test, the Object Assembly Test in the Wechsler Adult Intelligence Scale (Wechsler, 1981), and Poltrock and Brown's (1984) figure integration task. Indeed, one might also include the picture puzzles originally described by Healy and Fernald (1911). There are, however, several reasons for believing that our task is tapping quite distinct functions:

1. The original pictures were cut up into regular square pieces, and consequently the participants could not use the shape of the fragments to facilitate the composition of the figure, as in the three tests mentioned above. In other words, the use of square fragments reduces the role of perceptual support while maintaining similar demands upon imagery processes.

2. Unlike Hooper's test, for example, our test does not involve any object recognition component: The participants are always told the name of the object before attempting to solve the puzzle. The pictures were selected in order to facilitate the use of mental images of the different objects, on the basis of Snodgrass and Vandervart's (1980) assessment of image agreement and familiarity. These factors tend to minimize the problem of imagining the objects, while still allowing us to manipulate the active processing load by varying both the number of pieces and the visual complexity of the pictures.

3 . In our task, the pieces of the puzzles are always present in the participants' view. This reduces any passive memory load from the entire picture or the individual pieces, unlike Poltrock and Brown's task, where the pieces are presented one by one in sequence. Moreover, the pieces were not degraded perceptually in any way, and so our task probably taps different processes from those involved in the recognition of fragmented pictures such as Gollin figures (see, e.g., Cremer \& Zeef, 1987; Frazier \& Hoyer, 1992; Gollin, 1960; Salthouse \& Prill, 1988).

4. Performance in our task can be evaluated not simply in an all-or-none manner on each trial but in terms of the percentage of pieces correctly located. This increases the task's sensitivity in evaluating individual differences.

5 . In our task, the participants cannot touch or move the pieces. This prevents a simple perceptual matching of the pieces by means of trial and error and enhances the use of imaginal processing.

6. Finally, our task can incorporate the manipulation of a number of experimental variables (such as the number of fragments, the proportion of rotated pieces, and the visual complexity of the pictures) and the use of different response measures (such as the number of correct locations, the number of correct links, and the solution time). These considerations make this a valuable technique for both clinical evaluation and experimental investigations.

Nevertheless, it is important to establish that our task possesses construct validity as a measure of active visuospatial processing and is not simply tapping visuospatial processing in general or (as suggested by Experiment 3 ) working memory capacity in general. As part of our ongoing program of research, we have collected data from a large number of participants on a span version of the jigsaw-puzzle task and on several other procedures measuring the capacity for processing either verbal or visuospatial information in working memory (see also Vecchi \& Cornoldi, 1999). To conclude this article, we describe the results of a principal components analysis carried out on the performance of these participants on six working memory tasks.

\section{Method}

Participants. A total of 208 Italian participants were recruited from a variety of sources. They included university students, people in employment, and retired people, and to maximize the variability in their scores on the relevant measures, they included people in occupations that are highly dependent on visuospatial abilities (such as architects or designers) as well as people in occupations that are more dependent on verbal abilities (see Salthouse, 1991; Salthouse et al., 1990). Their ages ranged from 19 to 70 years, with a mean of 44.5 years.

Materials and Procedure. Six different tasks were used in this experiment: two verbal tasks and four visuospatial tasks. Three tasks were identified as being dependent upon active processing, and the other three were identified as being dependent upon passive processing. Each involved the presentation of a sequence of trials in an increasing order of difficulty until the participant was unable to solve at least two out of three items at any level. Performance on each task was evaluated by taking the mean value of the three most difficult items that had been solved. The order of presentation of the active and passive tasks was counterbalanced across the participants, and the order of presentation of the three tasks within each group followed a Latin-square design. The participants were allowed a break between the passive and active tasks, and the duration of the entire experiment was approximately $1 \mathrm{~h}$.

The listening span task was a version of the task devised by Daneman and Carpenter (1980) and adapted for use with Italian participants by De Beni, Palladino, Pazzaglia, and Cornoldi (1998). The participants were required to analyze a sequence of orally presented sentences while simultaneously memorizing the last word of each sentence. For example, the participants might be presented with the sentences, "Plumbers usually fix cars" and "A house is built with cement and bricks," and immediately after each sentence they had to judge whether it was true or false (i.e., "false" and "true," respectively). After their response to the last sentence, participants had to recall the final word in each sentence (i.e., cars, bricks). The number of sentences was increased until the participants could no longer correctly recall the sequence of final words. The score was the number of words in each correctly recalled sequence. This was regarded as a task that required active verbal processing.

The verbal span task was described by Spinnler and Tognoni (1987). Sequences consisting of disyllabic words were read aloud by the experimenter and had to be repeated by the participants immediately afterward. The number of words in each sequence was increased until it could no longer be correctly recalled. The score was again the number of words in each correctly recalled sequence. This was regarded as a task that required passive verbal processing.

For jigsaw puzzles, the enlarged pictures used in Experiments 1-3 were fragmented into $4,6,9,12$, and 16 identical pieces (that is, four pieces $7.5 \mathrm{~cm} \times 7.5 \mathrm{~cm}$, six pieces $5 \mathrm{~cm} \times 7.5 \mathrm{~cm}$, nine pieces $5 \mathrm{~cm}$ $\times 5 \mathrm{~cm}, 12$ pieces $5 \mathrm{~cm} \times 3.75 \mathrm{~cm}$, and 16 pieces $3.75 \mathrm{~cm} \times 3.75 \mathrm{~cm}$ ). The puzzles were presented as in Experiment 1, but with a progressively increasing processing load $(4,6,9,12$, and 16 fragments) until the puzzle could no longer be solved within a time limit of $150 \mathrm{sec}$. The score was the number of fragments in each correctly solved puz- 
zle, and this was regarded as a task that required active, simultaneous, visuospatial processing.

The mental pathways task was devised by Cornoldi et al. (1991; see also Vecchi et al., 1995). The participants were asked to imagine matrices containing different numbers of squares and to follow pathways among the squares in response to sequences of directions (left, right, forward, backward) that were read aloud by the experimenter. The starting square was always at the top left-hand corner of the matrix. A trial at Level 1 (practice) consisted of a $2 \times 2$ matrix with one statement, a trial at Level 2 consisted of a $2 \times 2$ matrix with two statements, a trial at Level 3 consisted of a $3 \times 3$ matrix with three statements, a trial at Level 4 consisted of a $3 \times 3$ matrix with five statements, a trial at Level 5 consisted of a $4 \times 4$ matrix with four statements, and so on. At the end of the sequence of instructions, the participants were asked to point to the final position in the imagined pathway on a blank matrix presented on a piece of paper. The score was the level of difficulty of each trial, and this was regarded as a task that required active, sequential, visuospatial processing.

Visual patterns was adapted from a task originally devised by Della Sala et al. (1997). Square matrices were devised that contained increasing numbers of squares, some of which were filled at random, leaving the remainder unfilled. Participants were instructed to memorize a matrix that was presented for $2 \mathrm{sec}$, and immediately afterward they were asked to reproduce the pattern on a blank matrix of the same size by identifying the filled squares. The score was the number of filled squares in each correctly recalled matrix, and this was regarded as a task that required passive, simultaneous, visuospatial processing.

Corsi blocks was originally devised by Corsi (1972; see also Milner, 1971; Spinnler \& Tognoni, 1987). The material consisted of nine wooden blocks arranged in random positions on a square board. The experimenter pointed to a particular sequence of blocks, and the participants had to reproduce that sequence immediately afterward. The number of blocks in each sequence was increased until it could no longer be correctly recalled. The score was the number of blocks in each correctly recalled sequence. This was regarded as a task that required passive, sequential, visuospatial processing because the participants had to recall a visuospatial sequence without carrying out any manipulation of the stored information.

\section{Results and Discussion}

A principal components analysis was carried out on the participants' scores on the six working memory tasks. This yielded three components with eigenvalues greater than one, and these explained $70.9 \%$ of the total variance in the data. The idea that three components should be extracted was confirmed by Cattell's (1966) scree test. These three components were then subjected to a varimax rotation, and the rotated solution is shown in Table 4.

The first principal component yielded significant loadings on listening span and verbal span. It can therefore be in- terpreted as a measure of verbal processing capacity. The second principal component exhibited significant loadings on jigsaw puzzles and mental pathways, and it can therefore be interpreted as a measure of active visuospatial processing capacity. The third principal component exhibited significant loadings on visual patterns and Corsi blocks, and it can therefore be interpreted as a measure of passive visuospatial processing capacity. It is of interest that the results of the principal components analysis reflect the processing demands of the different tasks (active vs. passive) and the modality in which information is presented (verbal vs. visual), but not the nature of that information (simultaneous vs. sequential).

The results of this analysis support the view that the jigsaw-puzzle task is specifically a measure of active visuospatial processing capacity. It showed no sign of any loading either on the component defined by the two verbal tasks or on the component defined by the two passive visuospatial tasks. This implies that manipulating the processing load by increasing the number of pieces in this task was not tapping the participants' general capacity for cognitive processing or their capacity for processing visuospatial information in any way whatsoever. Rather, it was selectively tapping their capacity for active visuospatial processing. Strictly speaking, the data obtained in Experiments 1-3 do not allow us to differentiate between effects of processing load of a general nature versus those of a selective nature. However, the present findings warrant the conclusion that the effects of processing load in those experiments were selective phenomena that specifically reflected the participants' limited capacity for active visuospatial processing.

However, the detailed results of the principal components analysis are equally important from a methodological point of view. It may be noted from Table 4 that the jigsawpuzzle task produced a much higher loading on the component that was identified with active visuospatial processing than the mental pathways test. Although the latter test should patently be regarded as a measure of active visuospatial processing, it seems legitimate to conclude that the jigsaw-puzzle test is to be preferred to the mental pathways test as a measure of that construct. The communality of the scores on the jigsaw-puzzle test in the principal components analysis was +.83 , and this can be interpreted as a conservative estimate of the reliability of the span version of this test (Pedhazur \& Schmelkin, 1991, p. 604). Thus,

Table 4

Loadings on Three Principal Components of Six Working Memory Tasks (Experiment 4)

\begin{tabular}{|c|c|c|c|c|}
\hline Task & Processing & 1 & 2 & 3 \\
\hline Listening span & Active, verbal & +.74 & +.02 & +.34 \\
\hline Verbal span & Passive, verbal & +.88 & +.10 & -.01 \\
\hline Jigsaw puzzles & Active, simultaneous, visuospatial & -.05 & +.91 & +.03 \\
\hline Mental pathways & Active, sequential, visuospatial & +.40 & +.58 & +.30 \\
\hline Visual patterns & Passive, simultaneous, visuospatial & +.14 & +.10 & +.91 \\
\hline Corsi blocks & Passive, sequential, visuospatial & +.15 & +.49 & +.50 \\
\hline
\end{tabular}

Note-Loadings greater than .50 in absolute magnitude are shown in bold. 
the results of this analysis support our earlier suggestions concerning the high level of reliability of the jigsaw-puzzle task.

One slightly anomalous feature of the solution shown in Table 4 is that the Corsi blocks test showed a marginally significant loading on the second component as well as a loading on the third component. For the purposes of this research, we construed this as a passive task, but, as mentioned earlier, its processing demands are in fact unclear (Berch et al., 1998). A number of studies have found that performance on the Corsi blocks test and other tasks requiring the short-term retention of sequences of spatial locations can be disrupted by concurrent tasks that demand active spatial attention or central executive control (Smyth \& Pelky, 1992; Smyth \& Scholey, 1994; Vecchi \& Richardson, 2001). This suggests that the short-term retention of sequences of spatial locations involves active visuospatial processing in working memory.

\section{GENERAL DISCUSSION}

The results obtained in the present investigation confirm that the jigsaw-puzzle task is a powerful, sensitive, and reliable tool for investigating visuospatial working memory in older people. In particular, the task appears to tap the functioning of the active elaboration component of VSWM, which has often been neglected in the research literature. The aim of developing an ecologically relevant procedure seems to have been successfully achieved: All the participants enjoyed carrying out the task, and they reported that their confidence was enhanced by the fact that they had carried out similar tasks in the past. The presentation of the correct picture at the end of each trial also helped to make the test enjoyable and relaxing. It has been stressed that environmental conditions are of great importance when assessing older people (see Baltes \& Baltes, 1990). Hence, our procedure seems to be especially appropriate for investigating the effects of individual differences related to chronological age.

The results of the present experiments have confirmed the findings of a previous study (Vecchi \& Richardson, 2000) suggesting that the active processing capacity of young adults is between six and nine operational units but depends on the overall complexity of the task. In the present investigation, the young adults produced better performance than the older adults in all of the conditions, and the older people appeared to be disproportionately affected by increased complexity. There was a monotonic effect of age, and the results could well be explained in terms of the notion that the reduced capacity of working memory affects cognitive performance in older people (e.g., Mayr \& Kliegl, 1993; Salthouse, 1994a). This reduction in processing capacity would produce effects upon both solution latency and solution accuracy of precisely the sort that were obtained here. Although the jigsaw-puzzle task taps visuospatial working memory processing as defined by Mayr and Kliegl (1993; Mayr et al., 1996), no evidence could be drawn from our data either to support or to reject the view that sequential complexity and coordinative complexity (Mayr \& Kliegl, 1993) are related to two different aspects of cognitive aging, as opposed to the view that a unique factor (i.e., speed of processing) affects performance in older people (Salthouse, 1994b, 1996). In short, the present findings are quite consistent with the hypothesis of a limited-capacity system that is vulnerable to increasing task complexity, whether due to an increase in the number of pieces or due to the rotation of some of the fragments.

The analysis of active processing capacity can be made more precise by examining the effects of visual complexity. A peculiar U-shaped effect became evident when the task became difficult and the active processing component was overloaded. In this case, the participants seemed to begin to use a perceptual strategy that was helpful in finding correct links among adjacent fragments. Visual complexity affected only the oldest participants in Experiment 1, but all three groups were affected in the more demanding task employed in Experiment 2. This perceptual effect could be regarded as the first signal that the system was becoming overloaded, even if this were not immediately reflected in the overall level of performance. It is, however, worth noting that the participants were not relying exclusively on the use of this perceptual strategy to carry out the task, since they always reported a higher number of correct locations than of correct links, a distinction that could be interpreted as a measure of the use of holistic versus perceptual strategies in carrying out the task.

Experiment 3 confirmed that this procedure could be adopted in the investigation of the working memory system. We showed that both specific visuospatial interference and a central interference disrupt performance to a similar extent. Though this result could also be explained by recent reformulations of Baddeley's traditional model of working memory (see, e.g., Logie \& Pearson, 1997) in terms of tasks requiring the integration of both specific and central processes, it seems more reasonable to distinguish between passive and active processes within the working memory system (see Cornoldi \& Vecchi, 2000). The jigsawpuzzle task was used in a recent study by Vecchi and Cornoldi (1999) to explore the idea of a selective deterioration of active working memory processes in old age. The authors confirmed that this task was specifically tapping the active component of working memory and that specific limitations could be found in these abilities in old age. Moreover, this study provides further confirmation that the discrepancies obtained by Salthouse and colleagues in assessing passive and active capacities in old age (Salthouse et al., 1991; Salthouse \& Mitchell, 1989) could have been determined by the choice of the experimental task and procedures: Vecchi and Cornoldi compared the levels of complexity of a passive visuospatial task and an active visuospatial task (the jigsaw-puzzle task), and older people were especially poor in the active task.

It should be emphasized that this effect of active processing is not simply an artifact of task complexity. This was shown in a recent study by Vecchi and Cornoldi (1999) in 
which task complexity was manipulated in active and passive tasks and performance was measured using a span procedure, as in Experiment 4: In each task, the span level reflected the highest level of complexity that the participants could deal with. The results supported the idea of a qualitative distinction between the passive storage and active processing components of working memory. Neuropsychological studies provide further confirmation of this hypothesis. Cornoldi, Rigoni, Venneri, and Vecchi (2000) described two children with nonverbal learning difficulties who showed a double dissociation between the ability to store visuospatial information and the ability to manipulate, transform, or integrate visuospatial material. Moreover, neuroimaging research has shown that different regions within the frontal lobes are activated in short-term storage and in the manipulation and transformation of information (Smith \& Jonides, 1999).

Thus, the importance of understanding the active component of visuospatial working memory - and, more generally, of the entire working memory system-is not restricted to the psychometric assessment of visuospatial functions or the analysis of individual differences in human cognition. As mentioned earlier, the distinction between a passive storage component and an active elaboration component of working memory is a key issue in the development of theoretical models of the structure and the function of human memory. Cornoldi and Vecchi (2000) recently suggested that the relationship between passive and active functions could be conceptualized along a continuum representing the overall balance between the storage and processing demands of a particular task. They devised a theoretical model of working memory in which each process was identified according to the nature of the material to be processed (verbal, visual, or spatial) and the nature of the cognitive manipulations required by the task. Nevertheless, recent research has shown that modality-specific effects can be obtained even in tasks that require high-level central processing (Shah \& Miyake, 1996), and this is also implied by the pattern of results shown in Table 4 . The experimental procedure that we have described seems to provide a means of studying the active visuospatial component of working memory.

In summary, the present investigation has not conclusively resolved the debate as to whether the passive and active components of VSWM are differentially affected by age (however, see Vecchi \& Cornoldi, 1999), but it has provided an extremely powerful tool for the investigation of the active component under ecologically relevant conditions. Performance varied with the overall complexity of this task, and older people exhibited a decline in their processing capacity. The fact that differences were obtained between younger and older old people in the present investigation suggests that the decline in the capacity for active elaboration is probably a lifelong process that continues throughout old age. Finally, within a working memory framework, we confirmed that this task involves the active manipulation of visuospatial information. This result is of particular importance since no other test of similar characteristics has as yet been developed. In contrast, the distinction between passive and active processes is turning out to be crucial, both in the development of theoretical models of memory functions and in the assessment of memory abilities in clinical and experimental practice.

\section{REFERENCES}

BADDELEY, A. D. (1986). Working memory. Oxford: Oxford University Press.

BADDELEY, A. D., \& HITCH, G. J. (1974). Working memory. In G. H. Bower (Ed.), The psychology of learning and motivation: Advances in research and theory (Vol. 8, pp. 47-90). New York: Academic Press.

Baltes, P. B., \& Baltes, M. M. (EDs.) (1990). Successful aging. Cambridge: Cambridge University Press.

Berch, D. B., Krikorian, R., \& Huha, E. M. (1998). The Corsi blocktapping task: Methodological and theoretical considerations. Brain \& Cognition, 38, 317-338.

Caplan, P. J., Crawford, M., Hyde, J. S., \& Richardson, J. T. E. (1997). Gender differences in human cognition. New York: Oxford University Press.

CARPEnTER, P. A., \& Just, M. A. (1989). The role of working memory in language comprehension. In D. Klahr \& K. Kotovsky (Eds.), Complex information processing: The impact of Herbert A. Simon (pp. 31-68). Hillsdale, NJ: Erlbaum.

CAttell, R. B. (1966). The scree test for the number of factors. Multivariate Behavioral Research, 1, 245-276.

Corballis, M. C., \& Beale, I. L. (1976). The psychology of left and right. Hillsdale, $\mathrm{NJ}$ : Erlbaum.

Cornoldi, C., Bertuccelli, B., Rocchi, P., \& Sbrana, B. (1993). Processing capacity limitations in pictorial and spatial representations in the totally congenitally blind. Cortex, 29, 675-689.

Cornoldi, C., Cortesi, A., \& Preti, D. (1991). Individual differences in the capacity limitations of visuospatial short-term memory: Research on sighted and totally congenitally blind people. Memory \& Cognition, 19, 459-468.

Cornoldi, C., Rigoni, F., Venneri, A., \& Vecchi, T. (2000). Passive and active processes in visuo-spatial memory: Double dissociation in developmental learning abilities. Brain \& Cognition, 43, 117-120.

Cornoldi, C., \& Vecchi, T. (2000). Mental imagery in blind people: The role of passive and active visuo-spatial processes. In M. Heller (Ed.), Touch, representation, and blindness (pp. 143-181). Oxford: Oxford University Press.

CoRsi, P. M. (1972). Human memory and the medial temporal region of the brain. Unpublished doctoral dissertation, McGill University, Montreal.

Craik, F. I. M., \& Salthouse, T. A. (Eds.) (1992). The handbook of aging and cognition. Hillsdale, $\mathrm{NJ}$ : Erlbaum.

Cremer, R., \& Zeef, E. J. (1987). What kind of noise increases with age? Journal of Gerontology, 42, 515-518.

Daneman, M., \& Carpenter, P. A. (1980). Individual differences in working memory and reading. Journal of Verbal Learning \& Verbal Behavior, 19, 450-466.

De Beni, R. Palladino, P., Pazzaglia, F., \& Cornoldi, C. (1998). Increases in intrusion errors and working memory deficit of poor comprehenders. Quarterly Journal of Experimental Psychology A, 51, 305-320.

Della Sala, S., Gray, C., Baddeley, A. D., \& Wilson, B. (1997). Visual pattern test. Bury St. Edmunds, U.K.: Thames Valley Test Co. DunNeTt, C. W. (1955). A multiple comparison procedure for comparing several treatments with a control. Journal of the American Statistical Association, 50, 1096-1121.

Farah, M. J., Hammond, K. M., Levine, D. N., \& Calvanio, R. (1988). Visual and spatial mental imagery: Dissociable systems of representation. Cognitive Psychology, 20, 439-462.

FraZIER,L., \& Hoy ER, W. J. (1992). Object recognition by component features: Are there age differences? Experimental Aging Research, 18, 9-14. GoLLIN, E. S. (1960). Developmental studies of visual recognition of incomplete objects. Perceptual \& Motor Skills, 11, 289-298. 
Harshman, R. A., Hampson, E., \& Berenbaum, S. A. (1983). Individual differences in cognitive abilities, Part 1: Sex and handedness differences in ability. Canadian Journal of Psychology, 37, 144-192.

Harshman, R. A., \& Paivio, A. (1987). "Paradoxical" sex differences in self-reported imagery. Canadian Journal of Psychology, 41, 287-302.

Healy, W., \& Fernald, G. M. (1911). Tests for practical mental classification. Psychological Monographs, 13(2, Whole No. 54), 1-53.

Helstrup, T. (1989). Active and passive memory: States, attitudes, and strategies. Scandinavian Journal of Psychology, 30, 113-133.

Hitch, G. J., Woodin, M. E., \& BAKer, S. (1989). Visual and phonological components of working memory in children. Memory \& Cognition, 17, 175-185.

Hooper, H. E. (1958). The Hooper visual organization test: Manual. Los Angeles: Western Psychological Services.

KERR, N. H. (1983). The role of vision in "visual imagery" experiments: Evidence from the congenitally blind. Journal of Experimental Psychology: General, 112, 265-277.

Kossly n, S. M. (1980). Image and mind. Cambridge, MA: Harvard University Press.

Kosslyn, S. M., Margolis, J. A., Barrett, A. M., Goldknopf, E. J., \& CAly, P. F. (1990). Age differences in imagery abilities. Child Development, 61, 995-1010.

LogIE, R. H. (1995). Visuospatialworking memory. Hove, U.K.: Erlbaum.

Logie, R. H., \& MARCHETTI, C. (1991). Visuospatial working memory: Visual, spatial or central executive. In R. H. Logie \& M. Denis (Eds.), Mental images in human cognition (pp. 105-115). Amsterdam: Elsevier.

Logie, R. H., \& Pearson, D. G. (1997). The inner eye and the inner scribe of visuospatial working memory: Evidence from developmental fractionation. European Journal of Cognitive Psychology, 9, 241-257.

Luzzatti, C., Vecchi, T., Agazzi, D., Cesa-Bianchi, M., \& Vergani, C. (1998). A neurological dissociation between visual and spatial processing in mental imagery. Cortex, 34, 461-469.

Maccoвy, E. E., \& JACKLIN, C. N. (1974). The psychology of sex differences. Stanford, CA: Stanford University Press.

Marmor, G., \& ZABACK, L. (1976). Mental rotation by the blind: Does mental rotation depend on visual imagery? Journal of Experimental Psychology: Human Perception \& Performance, 2, 515-521.

MaYr, U., \& KLIEGL, R. (1993). Sequential and coordinative complexity: Age-based processing limitations in figural transformations. Journal of Experimental Psychology: Learning, Memory, \& Cognition, 19, 1297-1320.

Mayr, U., Kliegl, R, \& Krampe, R. T. (1996). Sequential and coordinative processing dynamics in figural transformations across the life span. Cognition, 59, 61-90.

Milner, B. (1971). Interhemispheric differences in the localization of psychological processes in man. British Medical Bulletin, 27, 272 277.

Morris, R. G., Gick, M. L., \& Craik, F. I. M. (1988). Processing resources and age differences in working memory. Memory \& Cognition, 16, 362-366.

Paivio, A., \& Clark, J. M. (1991). Static versus dynamic imagery. In C. Cornoldi \& M. A. McDaniel (Eds.), Imagery and cognition (pp. 221-245). New York: Springer-Verlag.

Paivio, A., \& Harshman, R. A. (1983). Factor analysis of a questionnaire on imagery and verbal habits and skills. Canadian Journal of Psychology, 37, 461-483.

Pedhazur, E. J., \& Schmelkin, L. P. (1991). Measurement, design, and analysis: An integrated approach. Hillsdale, NJ: Erlbaum.

Poltrock, S. E., \& Brown, P. (1984). Individual differences in visual imagery and spatial ability. Intelligence, 8, 93-138.

Roberts, R. J., \& Aman, C. J. (1993). Developmental differences in giving directions: Spatial frames of reference and mental rotation. Child Development, 64, 1258-1270.

Salthouse, T. A. (1991). Theoretical perspectives on cognitive aging. Hillsdale, NJ: Erlbaum.
Salthouse, T. A. (1992). Reasoning and spatial abilities. In F. I. M. Craik \& T. A. Salthouse (Eds.), The handbook of aging and cognition (pp. 167-211). Hillsdale, NJ: Erlbaum.

Salthouse, T. A. (1994a). The aging of working memory. Neuropsychology, 8, 535-543.

SAlthouse, T. A. (1994b). The nature of the influence of speed on adult age differences in memory. Developmental Psychology, 30, 240-259.

SAlthouse, T. A. (1996). General and specific speed mediation of adult age differences in memory. Journal of Gerontology: Psychological Science B, 51, 30-42.

Salthouse, T. A., Baвсоск, R. L., \& Shaw, R. J. (1991). Effects of adult age on structural and operational capacities in working memory. Psychology \& Aging, 6, 118-127.

Salthouse, T. A., Babcock, R. L., Skovronek, E., Mitchell, D. R. D., \& Palmon, R. (1990). Age and experience effects in spatial visualization. Developmental Psychology, 26, 1228-1236.

Salthouse, T. A., \& Coon, E. J. (1993). Influence of task-specific processing speed on age differences in memory. Journal of Gerontology: Psychological Science, 48, 245-255.

Salthouse, T. A., \& Mitchell, D. R. D. (1989). Structural and operational capacities in integrative spatial ability. Psychology \& Aging, 4, 18-25.

Salthouse, T. A., \& Prill, K. A. (1988). Effects of aging on perceptual closure. American Journal of Psychology, 101, 217-238.

SHAH, P., \& MiYAKE, A. (1996). The separability of working memory resources for spatial thinking and language processing: An individual differences approach. Journal of Experimental Psychology: General, 125, 4-27.

Smith, E. E., \& Jonides, J. (1999). Storage and executive processes in the frontal lobes. Science, 283, 1657-1661.

Smyth, M. M., \& Pelky, P L. (1992). Short-term retention of spatial information. British Journal of Psychology, 83, 359-374.

Smyth, M. M., \& Scholey, K. A. (1994). Interference in immediate spatial memory. Memory \& Cognition, 22, 1-13.

SNOdGrass, J. G., \& VANDERWART, M. (1980). A standardized set of 260 pictures: Norms for name agreement, image agreement, familiarity, and visual complexity. Journal of Experimental Psychology: Human Learning \& Memory, 6, 174-215.

Spinnler, H., \& Tognoni, G. (EDS.) (1987). Standardizzazione e taratura italiana di test neuropsicologici [Italian standardization of new psychological tests]. Italian Journal of Neurological Science (Suppl. 8).

SWANSON, H. L. (1996). Individual and age-related differences in children's working memory. Memory \& Cognition, 24, 70-82.

VECCHI, T., (1998). Visuospatial limitations in congenitally totally blind people. Memory, 6, 91-102.

VECCHI, T., \& CoRNOLDI, C. (1999). Passive storage and active manipulation in visuo-spatial working memory: Further evidence from the study of age differences. European Journal of Cognitive Psychology, 11, 391-406.

Vecchi, T., \& Girelli, L. (1998). Gender differences in visuospatial processing: The importance of distinguishing between passive storage and active manipulation. Acta Psychologica, 99, 1-16.

VecChi, T., Monticelli, M. L., \& CoRnoldi, C. (1995). Visuospatial working memory: Structures and variables affecting a capacity measure. Neuropsychologia, 33, 1549-1564.

VECCHI, T., \& RichaRdSON, J. T. E. (2000). Active processing in visuospatial working memory. Cahiers de Psychologie Cognitive, 19, 3-32.

Vecchi, T., \& Richardson, J. T. E. (2001). Measures of visuospatial short-term memory: The Knox cube imitation test and the Corsi blocks test compared. Brain \& Cognition, 46, 91-94.

WECHSLER, D. (1981). Wechsler adult intelligent scale-Revised. New York: Psychological Corp.

Winer, B. J., Brown, D. R., \& Michels, K. M. (1991). Statistical principles in experimental design (3rd ed.). New York: McGraw-Hill. 


\section{APPENDIX}

\section{Stimulus Materials}

The mean ratings of each picture according to the norms of Snodgrass and Vanderwart (1980) are shown below on scales from 1 to 5, in which higher ratings represent greater visual complexity, greater familiarity, and greater agreement with one's own mental images, respectively.

\begin{tabular}{|c|c|c|c|}
\hline & $\begin{array}{c}\text { Visual } \\
\text { Complexity } \\
\end{array}$ & Familiarity & $\begin{array}{c}\text { Image } \\
\text { Agreement } \\
\end{array}$ \\
\hline \multicolumn{4}{|l|}{ Set VC1 } \\
\hline Lamp & 1.85 & 4.20 & 3.26 \\
\hline Chair & 2.05 & 4.58 & 3.22 \\
\hline Kettle & 2.40 & 3.86 & 3.31 \\
\hline Means & 2.10 & 4.21 & 3.26 \\
\hline \multicolumn{4}{|l|}{ Set VC2 } \\
\hline Toaster & 2.78 & 4.08 & 3.92 \\
\hline Watering can & 2.78 & 2.72 & 4.08 \\
\hline Chest of drawers & 2.95 & 4.52 & 3.22 \\
\hline Means & 2.84 & 3.77 & 3.74 \\
\hline \multicolumn{4}{|l|}{ Set VC3 } \\
\hline Television & 3.22 & 4.82 & 4.00 \\
\hline Iron & 3.25 & 3.68 & 4.08 \\
\hline Shoe & 3.38 & 4.62 & 3.02 \\
\hline Means & 3.28 & 4.37 & 3.70 \\
\hline \multicolumn{4}{|l|}{ Set VC4 } \\
\hline Watch & 3.40 & 4.58 & 3.18 \\
\hline Baby carriage (pram) & 3.42 & 2.72 & 3.65 \\
\hline Traffic light & 3.45 & 4.55 & 4.08 \\
\hline Means & 3.42 & 3.95 & 3.64 \\
\hline \multicolumn{4}{|l|}{ Set VC5 } \\
\hline Telephone & 3.52 & 4.80 & 4.28 \\
\hline Bicycle & 3.85 & 3.78 & 3.40 \\
\hline Motorcycle & 4.78 & 3.25 & 3.64 \\
\hline Means & 4.05 & 3.94 & 3.77 \\
\hline \multicolumn{4}{|l|}{$\begin{array}{l}\text { Practice items } \\
\quad \text { (Experiments } 1 \text { and } 2)\end{array}$} \\
\hline Clock & 2.68 & 4.38 & 2.20 \\
\hline Suitcase & 3.60 & 3.65 & 2.98 \\
\hline
\end{tabular}

(Manuscript received July 27, 1999; revision accepted for publication June 17, 2001.) 